Research Article

\title{
Some Random Fixed-Point Theorems for Weakly Contractive Random Operators in a Separable Banach Space
}

\author{
Kenza Benkirane, Abderrahim EL Adraoui $\left(\mathbb{D}\right.$, and El Miloudi Marhrani ${ }^{(D)}$
}

Faculty of Sciences Ben M'Sik, Hassan II University of Casablanca, Casablanca, Morocco

Correspondence should be addressed to Abderrahim EL Adraoui; a.adraoui@live.fr

Received 15 May 2021; Accepted 19 November 2021; Published 7 December 2021

Academic Editor: Nawab Hussain

Copyright (C) 2021 Kenza Benkirane et al. This is an open access article distributed under the Creative Commons Attribution License, which permits unrestricted use, distribution, and reproduction in any medium, provided the original work is properly cited.

The aim of this paper is to prove a common random fixed-point and some random fixed-point theorems for random weakly contractive operators in separable Banach spaces. A random Mann iterative process is introduced to approximate the fixed point. Finally, the main result is supported by an example and used to prove the existence and the uniqueness of a solution of a nonlinear stochastic integral equation system.

\section{Introduction}

The fixed-point theory has been revealed as a very powerful and important tool in the study of different mathematical models expressed in the forms of differential equations [1], integral equations [2, 3], fractional differential equations $[4,5]$, matrix equations [6], etc. Also, its applications are very useful and interesting in economics, in game theory, in computer science, and in other domains.

Probabilistic functional analysis is one of the essential mathematical disciplines that are applied to solving problems, characterized with uncertainties, known as probabilistic models. The random fixed-point theorems are stochastic generalizations of classical fixed-point theorems which are known as deterministic results and are required for the theory of random equations, random matrices, random partial differential equations, and various classes of random operators.

The theory of random fixed point was initiated by the Prague School of Probability in the 1950s. The random fixedpoint theory finds its roots in the work of Špaček [7] and Hanš $[8,9]$. They established a stochastic generalization of Banach contraction principle (BCP), and they applied their results to study the existence of a solution of random linear Fredholm integral equations. In 1976, Bharucha-Reid published his review article [10] which has attracted the attention of several researchers and which has led to the development of random fixed-point theory. In 1979, Itoh [11] extended Špaček's and Hanš’s theorems to multivalued contraction random mappings. The result obtained by Itoh in [11] was applied to solve a random differential equation in Banach space. In the recent past, random differential equations and random integral equations have been solved by random fixed-point theorems (see, for example, [12-16]). For some important contributions in the random fixedpoint theory, we invite the reader to consult [17-25] and the references therein.

It is necessary to mention that the BCP is the first fundamental deterministic fixed-point theorem in a metric space.

Theorem 1 (see [26]). If $(X, d)$ is a complete metric space and $T: X \longrightarrow X$ is a self-mapping such that

$$
\mathrm{d}(T x, T y) \leq k d(x, y),
$$

for all $x, y \in X$ and $k \in] 0,1[$, then $T$ has a unique fixed point.

Among the generalizations of this principle, we find the following theorem established in 2001 by Rhoades. 
Theorem 2 (see [27]). If $(X, d)$ is a complete metric space and $T: X \longrightarrow X$ is a self-mapping such that

$$
d(T x, T y) \leq d(x, y)-\phi(d(x, y))
$$

for all $x, y \in X$, and $\phi:[0, \infty[\longrightarrow[0, \infty[$ is a continuous and nondecreasing function such that $\phi(t)=0$ if and only if $t=0$, then $T$ has a unique fixed point.

Several interesting weak contractions were considered in various frameworks (see, for example, [28-31] and references therein). Among all these weak contractions, we are interested in the one studied by Eslamian and Abkar in [32]. We state the result in the following.

Theorem 3 (see [32]). If $(X, d)$ is a complete metric space and $T: X \longrightarrow X$ is a $\left(\psi_{1}, \psi_{2}, \varphi\right)$-weakly contractive selfmapping, i.e.,

$$
\psi_{1}(d(T(x), T(y))) \leq \psi_{2}(d(x, y))-\varphi(d(x, y)) \text {, for all } x, y \in X,
$$

then $T$ has a unique fixed point.Here, the three functions $\psi_{1}, \psi_{2}, \varphi: \mathbb{R}^{+} \longrightarrow \mathbb{R}^{+}$, called control functions, satisfy the following conditions:
(a) $\psi_{1}$ and $\psi_{2}$ are continuous
(b) $\varphi$ is lower semicontinuous
(c) $\psi_{1}$ is increasing
(d) For $i \in\{1,2\}, \psi_{i}(t)=0$ if and only if $t=0$
(e) For all $t \in \mathbb{R}^{+}, \varphi(t)=0$ if and only if $t=0$
(f) For all $t>0, \psi_{1}(t)-\psi_{2}(t)+\varphi(t)>0$

In this study, we prove a common random fixed-point theorem and some random fixed-point theorems for random $\left(\psi_{1}, \psi_{2}, \varphi\right)$-weakly contractive operators in a separable Banach space, where the three control functions $\psi_{1}, \psi_{2}$, and $\varphi$ satisfy all the conditions $(a)-(f)$ except the condition $(d)$ which is replaced by the weak condition:

$$
\psi_{2}(t)=0 \quad \text { if and only if } t=0 \text {. }
$$

As an application, we show the existence and the uniqueness of a random solution for a system of nonlinear integral equations. To prove our main results, we need to recall the following concepts and results. For more details, the reader may consult $[33,34]$.

\section{Preliminaries}

Let $(X,\|\|$.$) be a separable Banach space, \beta_{X}$ be the $\sigma$-algebra of all Borel subsets of $X$, and $(\Omega, \beta, \mu)$ be a complete probability measure space with the measure $\mu$ and $\beta$ be the $\sigma$-algebra of subsets of $\Omega$. Let $C$ be a nonempty subset of $X$.

Definition 1 (see [33, 35]).

(i) A mapping $x: \Omega \longrightarrow X$ is said to be a random variable with values in $X$ if the inverse image under the mapping $x$ of every Borel set $B$ of $X$ belongs to $\beta$, that is, $x^{-1}(B) \in \beta$ for all $B \in \beta_{X}$

(ii) A mapping $x: \Omega \longrightarrow C$ is called a random variable with values in $C$ if $x^{-1}(B \cap C) \in \beta$ for all $B \in \beta_{X}$

Definition 2 (see [33, 34]).

(i) A mapping $x: \Omega \longrightarrow X$ is said to be a finitely-valued random variable if it is constant on each of a finite number of disjoint sets $A_{i} \in \beta$ and equal to 0 on $\Omega-\left(\cup_{i=1}^{n} A_{i}\right)$.

(ii) A mapping $x: \Omega \longrightarrow X$ is said to be a simple random variable if it is finitely valued.

(iii) A mapping $x: \Omega \longrightarrow X$ is said to be a strong (or Bochner) random variable if there exists a sequence $\left(x_{n}\right)_{n}$ of simple random variables which converges to $x$ almost surely, that is, there exists a set $A_{0} \in \beta$ with $\mu\left(A_{0}\right)=0$ such that

$\lim _{n \rightarrow \infty}\left\|x_{n}(\omega)-x(\omega)\right\|=0 \quad$ for every $\omega \in \Omega-A_{0}$.

Next, we introduce the notion of a weak random variable.

Definition 3 (see [33]). A mapping $x: \Omega \longrightarrow X$ is said to be a weak (or Pettis) random variable if the functions $x^{*}(x)$ are real-valued random variables for each $x^{*} \in X^{*}$, where $X^{*}$ denotes the first normed dual space of $X$.

Remark 1 (see [33]). In this work, we restrict our attention to the case where $X$ is a separable Banach space. In this setting, the concept of weak and strong random variables is equivalent.

The following definition of the mode of convergence for Banach space-valued random variables, which we use in the sequel, is borrowed from [33].

Definition 4. Let $\left(x_{n}\right)_{n}$ and $x$ be $X$-valued random variables. The sequence $\left(x_{n}\right)_{n}$ converges to $x$ in $\Omega$ strongly almost surely if there exists a set $A_{0} \in \beta$ with $\mu\left(A_{0}\right)=0$ such that

$\lim _{n \longrightarrow \infty}\left\|x_{n}(\omega)-x(\omega)\right\|=0 \quad$ for any $\omega \in \Omega-A_{0}$.

We recall the following results from the study by Joshi and Bose ([34], Theorem 6.1.2).

Theorem 4. Let $x$ and $y$ be two strong random variables and $\alpha$ and $\beta$ be two constants. Then, the following statements hold:

(i) $\alpha x+\beta y$ is a strong random variable

(ii) If $f$ is a real-valued random variable and $x$ is a strong random variable, then $f x$ is a strong random variable

(iii) If $\left(x_{n}\right)_{n}$ is a sequence of strong random variables converging strongly to $x$ almost surely, then $x$ is a strong random variable 
Definition 5 (see [35]).

(i) A mapping $T: \Omega \times C \longrightarrow C$ is said to be random operator if for each $x \in C$, the mapping $T(., x): \Omega \longrightarrow C$ is measurable

(ii) A random operator $T: \Omega \times C \longrightarrow C$ is continuous if the set of all $\omega \in \Omega$ for which $T(\omega,$.$) is continuous$ has measure one

Throughout this paper, we denote $\mathscr{R} V(X)$ as the set of all $X$-valued random variables and we adopt the following definition of the random fixed point given by Joshi and Bose in [34].

Definition 6. Let $x \in \mathscr{R} V(X) . x$ is said to be a random fixed point of $T$ if $\mu\{\omega: T(\omega, x(\omega))=x(\omega)\}=1$.

\section{Main Results}

In this section, we prove a common random fixed-point theorem and some random fixed-point theorems for $\left(\psi_{1}, \psi_{2}, \varphi\right)$-weakly contractive mappings in a separable Banach space.

Definition 7. Let $(X,\|\|$.$) be a separable Banach space and$ $(\Omega, \beta, \mu)$ be a complete probability measure space. The mapping $T: \Omega \times X \longrightarrow X$ is called a $\left(\psi_{1}, \psi_{2}, \varphi\right)$-weakly contractive random operator if $T$ satisfies the following inequality:

$$
\psi_{1}\left(\left\|T\left(\omega, x_{1}(\omega)\right)-T\left(\omega, x_{2}(\omega)\right)\right\|\right) \leq \psi_{2}\left(\left\|x_{1}(\omega)-x_{2}(\omega)\right\|\right)-\varphi\left(\left\|x_{1}(\omega)-x_{2}(\omega)\right\|\right),
$$

almost surely, for all $x_{1}, x_{2} \in \mathscr{R} V(X)$.

Theorem 5. Let $(X,\|\|$.$) be a separable Banach space, C$ be a nonempty closed subset of $X$, and $(\Omega, \beta, \mu)$ be a complete probability measure space. Let $T, S: \Omega \times C \longrightarrow C$ be two continuous random operators satisfying

$$
\psi_{1}\left(\left\|T\left(\omega, x_{1}(\omega)\right)-S\left(\omega, x_{2}(\omega)\right)\right\|\right) \leq \psi_{2}\left(\left\|x_{1}(\omega)-x_{2}(\omega)\right\|\right)-\varphi\left(\left\|x_{1}(\omega)-x_{2}(\omega)\right\|\right)
$$

almost surely, for all $x_{1}, x_{2} \in \mathscr{R} V(C)$. Then, there exists a unique common random fixed point of $S$ and $T$.

Proof. Let $x_{0} \in \mathscr{R} V(C)$. Let $x_{1}(\omega)=S\left(\omega, x_{0}(\omega)\right)$ and $x_{2}(\omega)=T\left(\omega, x_{1}(\omega)\right)$. Since $S$ and $T$ are two continuous random operators in a separable space, it follows that $x_{1}$ and $x_{2}$ are in $\mathscr{R} V(C)$. Now, consider the sequence defined, for each $n \in \mathbb{N}$, by $x_{2 n+1}(\omega)=S\left(\omega, x_{2 n}(\omega)\right) \quad$ and $x_{2 n+2}(\omega)=T\left(\omega, x_{2 n+1},(\omega)\right)$.
By induction, $\left(x_{n}\right)_{n}$ is a sequence in $\mathscr{R} V(C)$. Consider the set $A=X \cap Y$ such that

$$
\begin{aligned}
& X=\{\omega \in \Omega: x \longrightarrow T(\omega, x) \text { is continuous on } C\}, \\
& Y=\{\omega \in \Omega: x \longrightarrow S(\omega, x) \text { is continuous on } C\} .
\end{aligned}
$$

For all $y, z \in \mathscr{R} V(C)$, we denote by $E_{y, z}$ the set of elements $\omega \in \Omega$ such that

$$
\psi_{1}(\|T(\omega, y(\omega))-S(\omega, z(\omega))\|) \leq \psi_{2}(\|y(\omega)-z(\omega)\|)-\varphi(\|y(\omega)-z(\omega)\|)
$$

Let $M=\bigcap_{n \in \mathbb{N}^{*}}\left(A \cap E_{x_{n}, x_{n+1}}\right)$. As stated, $\mu(A)=1$ and $\mu\left(E_{x_{n}, x_{n+1}}\right)=1$, for each $n \in \mathbb{N}^{*}$. Then, $\mu(M)=1$. Let $\omega \in M$.

For all $n \in \mathbb{N}^{*}$, we have

$$
\psi_{1}\left(\left\|x_{n+1}(\omega)-x_{n}(\omega)\right\|\right) \leq \psi_{2}\left(\left\|x_{n}(\omega)-x_{n-1}(\omega)\right\|\right)-\varphi\left(\left\|x_{n}(\omega)-x_{n-1}(\omega)\right\|\right) .
$$

This implies that 
$\left\|x_{n+1}(\omega)-x_{n}(\omega)\right\| \leq\left\|x_{n}(\omega)-x_{n-1}(\omega)\right\|, \quad$ for all $n \in \mathbb{N}^{*}$

Indeed, let us assume that there exists $n_{0} \in \mathbb{N}^{*}$ such that

$$
\left\|x_{n_{0}+1}(\omega)-x_{n_{0}}(\omega)\right\|>\left\|x_{n_{0}}(\omega)-x_{n_{0}-1}(\omega)\right\| .
$$

Since $\psi_{1}$ is increasing, we get

$$
\psi_{1}\left(\left\|x_{n_{0}+1}(\omega)-x_{n_{0}}(\omega)\right\|\right)>\psi_{1}\left(\left\|x_{n_{0}}(\omega)-x_{n_{0}-1}(\omega)\right\|\right) .
$$

Then,

$$
\psi_{1}\left(\left\|x_{n_{0}+1}(\omega)-x_{n_{0}}(\omega)\right\|\right)>\psi_{2}\left(\left\|x_{n_{0}}(\omega)-x_{n_{0}-1}(\omega)\right\|\right)-\varphi\left(\left\|x_{n_{0}}(\omega)-x_{n_{0}-1}(\omega)\right\|\right),
$$

which is a contradiction. Hence, for all $n \in \mathbb{N}^{*}$, we have

$$
\lim _{n \longrightarrow \infty}\left\|x_{n+1}(\omega)-x_{n}(\omega)\right\|=l .
$$

$$
\left\|x_{n+1}(\omega)-x_{n}(\omega)\right\| \leq\left\|x_{n}(\omega)-x_{n-1}(\omega)\right\| .
$$

Since

It follows that the sequence $\left(\left\|x_{n+1}(\omega)-x_{n}(\omega)\right\|\right)_{n}$ is decreasing and consequently there exists $l \geq 0$ such that

$$
\begin{aligned}
& \limsup _{n \longrightarrow+\infty} \psi_{1}\left(\left\|x_{n+1}(\omega)-x_{n}(\omega)\right\|\right) \\
& \leq \limsup _{n \longrightarrow+\infty}\left(\psi_{2}\left(\left\|x_{n}(\omega)-x_{n-1}(\omega)\right\|\right)-\varphi\left(\left\|x_{n}(\omega)-x_{n-1}(\omega)\right\|\right)\right) \\
& \leq \limsup _{n \longrightarrow+\infty} \psi_{2}\left(\left\|x_{n}(\omega)-x_{n-1}(\omega)\right\|\right)-\liminf _{n \longrightarrow+\infty} \varphi\left(\left\|x_{n}(\omega)-x_{n-1}(\omega)\right\|\right)
\end{aligned}
$$

and by using the continuity of $\psi_{1}$ and $\psi_{2}$ and the lower semicontinuity of $\varphi$, we obtain that $\psi_{1}(l) \leq \psi_{2}(l)-\varphi(l)$, which is a contradiction unless $l=0$.

Hence,

$$
\lim _{n \longrightarrow \infty}\left\|x_{n+1}(\omega)-x_{n}(\omega)\right\|=0, \quad \text { for all } \omega \in M .
$$

Now, fix $\omega$ in $M$ and let us prove that $\left(x_{n}(\omega)\right)_{n}$ is a Cauchy sequence in $C$. For this, it is sufficient to show that the subsequence $\left(x_{2 n}(\omega)\right)_{n}$ is a Cauchy sequence. If we assume the contrary, then

$$
\exists \int>0, \forall k \in \mathbb{N}, \exists m_{k}>n_{k} \geq k \quad \text { such that }\left\|x_{2 m_{k}}(\omega)-x_{2 n_{k}}(\omega)\right\| \geq \int
$$

Furthermore, corresponding to $n_{k}$, we can choose $m_{k}$ in such a way that it is the smallest integer with $m_{k}>n_{k}$ satisfying

$$
\left\|x_{2 m_{k}}(\omega)-x_{2 n_{k}}(\omega)\right\| \geq \int .
$$

Consequently,

$$
\left\|x_{2 m_{k}-2}(\omega)-x_{2 n_{k}}(\omega)\right\|<\int .
$$

By using the triangular inequality, we obtain

$$
\begin{aligned}
\epsilon & \leq\left\|x_{2 m_{k}}(\omega)-x_{2 n_{k}}(\omega)\right\| \leq\left\|x_{2 m_{k}}(\omega)-x_{2 m_{k}-1}(\omega)\right\|+\left\|x_{2 m_{k}-1}(\omega)-x_{2 n_{k}}(\omega)\right\| \\
\leq & \left\|x_{2 m_{k}}(\omega)-x_{2 m_{k}-1}(\omega)\right\|+\left\|x_{2 m_{k}-1}(\omega)-x_{2 m_{k}-2}(\omega)\right\| \\
& \quad+\left\|x_{2 m_{k}-2}(\omega)-x_{2 n_{k}}(\omega)\right\| \\
& <\left\|x_{2 m_{k}}(\omega)-x_{2 m_{k}-1}(\omega)\right\|+\left\|x_{2 m_{k}-1}(\omega)-x_{2 m_{k}-2}(\omega)\right\|+\epsilon .
\end{aligned}
$$


Hence,

Also,

$$
\lim _{k \longrightarrow \infty}\left\|x_{2 m_{k}}(\omega)-x_{2 n_{k}}(\omega)\right\|=\varepsilon
$$

$$
\begin{aligned}
& \left\|x_{2 m_{k}}(\omega)-x_{2 n_{k}}(\omega)\right\|-\left\|x_{2 m_{k}+1}(\omega)-x_{2 m_{k}}(\omega)\right\| \leq\left\|x_{2 m_{k}+1}(\omega)-x_{2 n_{k}}(\omega)\right\| \\
& \left\|x_{2 m_{k}+1}(\omega)-x_{2 n_{k}}(\omega)\right\| \leq\left\|x_{2 m_{k}+1}(\omega)-x_{2 m_{k}}(\omega)\right\|+\left\|x_{2 m_{k}}(\omega)-x_{2 n_{k}}(\omega)\right\|
\end{aligned}
$$

Letting $k \longrightarrow+\infty$, we obtain

$$
\lim _{k \longrightarrow \infty}\left\|x_{2 m_{k}+1}(\omega)-x_{2 n_{k}}(\omega)\right\|=\epsilon
$$

$$
\begin{aligned}
& \left\|x_{2 m_{k}}(\omega)-x_{2 n_{k}-1}(\omega)\right\| \leq\left\|x_{2 n_{k}-1}(\omega)-x_{2 n_{k}}(\omega)\right\|+\left\|x_{2 m_{k}}(\omega)-x_{2 n_{k}}(\omega)\right\|, \\
& \left\|x_{2 m_{k}}(\omega)-x_{2 n_{k}}(\omega)\right\|-\left\|x_{2 n_{k}-1}(\omega)-x_{2 n_{k}}(\omega)\right\| \leq\left\|x_{2 m_{k}}(\omega)-x_{2 n_{k}-1}(\omega)\right\| .
\end{aligned}
$$

Then,

We have, for all $k \in \mathbb{N}$,

$$
\lim _{k \longrightarrow \infty}\left\|x_{2 m_{k}}(\omega)-x_{2 n_{k}-1}(\omega)\right\|=\int
$$

$$
\left.\psi_{1}\left(\| x_{2 m_{k}+1}\right)(\omega)-x_{2 n_{k}}(\omega) \|\right)=\psi_{1}\left(\left\|S\left(\omega, x_{2 m_{k}}(\omega)\right)-T\left(\omega, x_{2 n_{k}-1}(\omega)\right)\right\|\right) \leq \psi_{2}\left(\left\|x_{2 m_{k}}(\omega)-x_{2 n_{k}-1}(\omega)\right\|\right)-\varphi\left(\left\|x_{2 m_{k}}(\omega)-x_{2 n_{k}-1}(\omega)\right\|\right)
$$

By passing to the upper limit, we get $\psi_{1}(\epsilon) \leq \psi_{2}(\epsilon)-\varphi(\epsilon)$, which is a contradiction since $\epsilon>0$. This shows that $\left\{\left(x_{2 n}(\omega)\right)_{n}\right\}$ is a Cauchy sequence in $C$, for each $\omega \in M$. Using (20), it is easy to check that $\left(x_{n}(\omega)\right)_{n}$ is a Cauchy sequence in $C$, for each $\omega \in M$.

Since $C$ is a closed subset of the Banach space $X$, then $C$ is complete, which implies that, for all $\omega \in M$, the sequence $\left(x_{n}(\omega)\right)_{n}$ converges by norm in $C$. Let $x: \Omega \longrightarrow C$ be the mapping such that $x(\omega)=\lim x_{n}(\omega)$, for each $\omega \in M$. Since the sequence $\left(x_{n}\right)_{n}$ converges strongly almost surely to $x$, then, according to ([33], Theorem 1.6), $x$ is a $C$-valued random variable.

Let $\omega \in M$. For all $n \in \mathbb{N}$, we have $x_{2 n+1}=S\left(\omega, x_{2 n}(\omega)\right)$ and $x_{2 n+2}=T\left(\omega, x_{2 n+1}(\omega)\right)$.

Since $x(\omega)=\lim x_{n}(\omega)$ and by using the continuity of $S$ and $T$, we get $S(\omega, x(\omega))=T(w, x(\omega))=x(\omega)$, for each $\omega \in M$.

Hence,

$\mu(\{\omega \in \Omega: S(\omega, x(\omega))=T(w, x(\omega))=x(\omega)\})=1$.
It means that $x$ is a common random fixed point of $S$ and $T$.

To prove the uniqueness of this common fixed point, let $y$ be another common random fixed point of $S$ and $T$. Consider the two sets

$$
\begin{aligned}
& F_{x}=\{\omega \in \Omega: S(\omega, x(\omega))=T(w, x(\omega))=x(\omega)\} \\
& F_{y}=\{\omega \in \Omega: S(\omega, y(\omega))=T(w, y(\omega))=y(\omega)\}
\end{aligned}
$$

Then, $\mu\left(F_{x} \cap F_{y} \cap M\right)=1$. Let $N=F_{x} \cap F_{y} \cap M$. For each $\omega \in N$, we have

$$
\begin{array}{r}
\psi_{1}(\|x(\omega)-y(\omega)\|)=\psi_{1}(\|T(\omega, x(\omega))-S(\omega, y(\omega))\|) \\
\leq \psi_{2}(\|x(\omega)-(y(\omega))\|-\varphi(\| x(\omega)-y(\omega)) \|) .
\end{array}
$$

This implies that $\|x(\omega)-y(\omega)\|=0$. Therefore, $x=y$ almost surely. This proves the uniqueness of the common random fixed point of $S$ and $T$. 
If $T=S$ in Theorem 5, we obtain the following random fixed-point theorem for $\left(\psi_{1}, \psi_{2}, \varphi\right)$-weakly contractive random mapping.

Corollary 1. Let $(X,\|\|$.$) be a separable Banach space, C$ be a nonempty closed subset of $X$, and $(\Omega, \beta, \mu)$ be a complete probability measure space. Let $T: \Omega \times C \longrightarrow C$ be a continuous $\left(\psi_{1}, \psi_{2}, \varphi\right)$-weakly contractive random mapping. Then, there exists a unique random fixed point of $T$.

Example 1. Let $X=\mathbb{R}^{2}$ with the norm 1 defined, for all $(x, y) \in \mathbb{R}^{2}$, as follows:

$$
\|(x, y)\|_{1}=|x|+|y| .
$$

$\Omega=[0,1]$ and $C=[0,1] \times[0,1]$. Let $\beta$ be a $\sigma$-algebra of Lebesgue measurable subsets of $\mathbb{R}^{2}$. Consider the three functions $\psi_{1}, \psi_{2}, \varphi: \leq[0,+\infty[\longrightarrow[0+\infty[$ defined for all $t \geq 0$ as follows:

$$
\psi_{1}(t)=t, \psi_{2}(t)=\frac{2 t}{3} \text { and } \varphi(t)= \begin{cases}\frac{t}{6} & \text { if } 0 \leq t \leq 2 \\ 1 & \text { if } t>2\end{cases}
$$

Consider the random operator $T: \Omega \times C \longrightarrow C$ defined by $T(\omega, x(\omega))=T\left(\omega, x_{1}(\omega), x_{2}(\omega)\right)$ $=\left(x_{1}(\omega)+\omega / 3 ; x_{1}(\omega)+\omega / 3\right)$, where $\omega \in \Omega \quad$ and $x=\left(x_{1}, x_{2}\right) \in \mathscr{R} V(C)$.

Let $\omega \in \Omega$. We have

$$
\begin{aligned}
& \psi_{1}\left(\left\|T\left(\omega, x_{1}(\omega), x_{2}(\omega)\right)-T\left(\omega, y_{1}(\omega), y_{2}(\omega)\right)\right\|_{1}\right) \\
& =\left\|T\left(\omega, x_{1}(\omega), x_{2}(\omega)\right)-T\left(\omega, y_{1}(\omega), y_{2}(\omega)\right)\right\|_{1}, \\
& =\left\|\left(\frac{x_{1}(\omega)+\omega}{3} ; \frac{x_{2}(\omega)+\omega}{3}\right)-\left(\frac{y_{1}(\omega)+\omega}{3} ; \frac{y_{2}(\omega)+\omega}{3}\right)\right\|_{1}, \\
& =\left\|\left(\frac{x_{1}(\omega)+\omega}{3}-\frac{y_{1}(\omega)+\omega}{3} ; \frac{x_{2}(\omega)+\omega}{3}-\frac{y_{2}(\omega)+\omega}{3}\right)\right\|_{1}, \\
& =\left\|\left(\frac{x_{1}(\omega)-y_{1}(\omega)}{3} ; \frac{x_{2}(\omega)-y_{2}(\omega)}{3}\right)\right\|_{1}, \\
& =\frac{\left|x_{1}(\omega)-y_{1}(\omega)\right|+\left|x_{2}(\omega)-y_{2}(\omega)\right|}{3} . \\
& \psi_{2}\left(\left\|\left(x_{1}(\omega) ; x_{2}(\omega)\right)-\left(y_{1}(\omega) ; y_{2}(\omega)\right)\right\|_{1}\right)=\psi_{2}\left(\left\|\left(\left[x_{1}(\omega)-y_{1}(\omega)\right] ;\left[x_{2}(\omega)-y_{2}(\omega)\right]\right)\right\|_{1}\right), \\
& =\psi_{2}\left(\left|x_{1}(\omega)-y_{1}(\omega)\right|+\left|x_{2}(\omega)-y_{2}(\omega)\right|\right) \text {, } \\
& =2 \frac{\left|x_{1}(\omega)-y_{1}(\omega)\right|+\left|x_{2}(\omega)-y_{2}(\omega)\right|}{3} \text {. } \\
& \varphi\left(\left\|\left(x_{1}(\omega) ; x_{2}(\omega)\right)-\left(y_{1}(\omega) ; y_{2}(\omega)\right)\right\|_{1}\right)=\varphi\left(\left\|\left(\left[x_{1}(\omega)-y_{1}(\omega)\right] ;\left[x_{2}(\omega)-y_{2}(\omega)\right]\right)\right\|_{1}\right), \\
& =\varphi\left(\left|x_{1}(\omega)-y_{1}(\omega)\right|+\left|x_{2}(\omega)-y_{2}(\omega)\right|\right) \text {, } \\
& =\frac{\left|x_{1}(\omega)-y_{1}(\omega)\right|+\left|x_{2}(\omega)-y_{2}(\omega)\right|}{6} \text {. }
\end{aligned}
$$

Then, 


$$
\begin{aligned}
\psi_{2}\left(\left\|\left(x_{1}(\omega) ; x_{2}(\omega)\right)-\left(y_{1}(\omega) ; y_{2}(\omega)\right)\right\|_{1}\right)-\varphi( & \left.\left\|\left(x_{1}(\omega) ; x_{2}(\omega)\right)-\left(y_{1}(\omega) ; y_{2}(\omega)\right)\right\|_{1}\right) \\
= & 2 \frac{\left|x_{1}(\omega)-y_{1}(\omega)\right|+\left|x_{2}(\omega)-y_{2}(\omega)\right|}{3}, \\
& -\frac{\left|x_{1}(\omega)-y_{1}(\omega)\right|+\left|x_{2}(\omega)-y_{2}(\omega)\right|}{6}, \\
& =\frac{\left|x_{1}(\omega)-y_{1}(\omega)\right|+\left|x_{2}(\omega)-y_{2}(\omega)\right|}{2}, \\
& \geq \frac{\left|x_{1}(\omega)-y_{1}(\omega)\right|+\left|x_{2}(\omega)-y_{2}(\omega)\right|}{3} .
\end{aligned}
$$

Consequently, for all $x=\left(x_{1} ; x_{2}\right), y=\left(y_{1} ; y_{2}\right) \in \mathscr{R} V$

$(C)$ and for each $\omega \in \Omega$,

$$
\psi_{1}\left(\|T(\omega, x(\omega))-T(\omega, y(\omega))\|_{1}\right)\left(\leq \psi_{2}\|x(\omega)-y(\omega)\|_{1}\right)-\varphi\left(\|x(\omega)-y(\omega)\|_{1}\right) .
$$

All conditions of Corollary 1 are satisfied and $T$ has a random fixed point which is

$$
x: \omega \longrightarrow\left(\frac{\omega}{2} ; \frac{\omega}{2}\right) \text {. }
$$

In Corollary 1 , if $\psi_{1}(t)=\psi_{2}(t)=t$, for all $t \geq 0$, we obtain the following corollary which is an improvement of ([36], Theorem 5.2) in a separable Banach space.
Corollary 2. Let $(X,\|\|$.$) be a separable Banach space, C$ be a nonempty closed subset of $X$, and $(\Omega, \beta, \mu)$ be a complete probability measure space. Let $T: \Omega \times C \longrightarrow C$ be a continuous random operator satisfying

$$
\left\|T\left(\omega, x_{1}(\omega)-T\left(\omega, x_{2}(\omega)\right)\right)\right\| \leq\left\|x_{1}(\omega)-x_{2}(\omega)\right\|-\varphi\left(\left\|x_{1}(\omega)-x_{2}(\omega)\right\|\right),
$$

almost surely, for all $x_{1}, x_{2} \in \mathscr{R} V(C)$. Then, there exists a unique random fixed point of $T$.

In Corollary 1 , if $\psi_{1}=\psi_{2}$, we obtain the following random fixed-point theorem for $(\psi, \varphi)$-weakly contractive random mapping.
Corollary 3. Let $(X,\|\|$.$) be a separable Banach space, C$ be a nonempty closed subset of $X$, and $(\Omega, \beta, \mu)$ be a complete probability measure space. Let $T: \Omega \times C \longrightarrow C$ be a continuous random operator satisfying the following condition:

$$
\psi\left(\left\|T\left(\omega, x_{1}(\omega)\right)-T\left(\omega, x_{2}(\omega)\right)\right\|\right) \leq \psi\left(\left\|x_{1}(\omega)-x_{2}\right\|(\omega)\right)-\varphi\left(\left\|x_{1}(\omega)-x_{2}(\omega)\right\|\right)
$$

almost surely, for all $x_{1}, x_{2} \in \mathscr{R} V(C)$. Then, there exists a unique random fixed point of $T$.

In Corollary 1 , if $\psi_{1}(t)=t$ and $\left(\psi_{2}-\varphi\right)(t)=k t$, for all $t \geq 0$ and for some $k \in[0,1$ [, we obtain the following random fixed-point theorem for Banach's contraction.

Corollary 4. Let $(X,\|\|$.$) be a separable Banach space, C$ be a nonempty closed subset of $X$, and $(\Omega, \beta, \mu)$ be a complete probability measure space. Let $T: \Omega \times C \longrightarrow C$ be a continuous random operator satisfying the following condition:

$$
\left\|T\left(\omega, x_{1}(\omega)\right)-T\left(\omega, x_{2}(\omega)\right)\right\| \leq k\left\|x_{1}(\omega)-x_{2}(\omega)\right\|,
$$

almost surely, for all $x_{1}, x_{2} \in \mathscr{R} V(C)$ and $k \in[0,1[$. Then, there exists a unique random fixed point of $T$.

\section{Random Mann Iteration Scheme}

In the following, we investigate the convergence of random Mann iteration scheme applied to a $\left(\psi_{1}, \psi_{2}, \varphi\right)$-weakly contractive random operator. 
Definition 8. (random Mann iteration scheme [35]). Let $T: \Omega \times C \longrightarrow C$ be a random operator, where $C$ is a nonempty convex subset of a separable Banach space $X$. A random Mann iteration scheme is the sequence of $C$-valued random variables $\left(x_{n}\right)_{n}$ defined, for all $\omega \in \Omega$, by

$$
\left\{\begin{array}{l}
x_{n+1}(\omega)=\left(1-c_{n}\right) x_{n}(\omega)+c_{n} T\left(\omega, x_{n}(\omega)\right), \quad \text { for all } n \in \mathbb{N} \\
x_{0}: \Omega \longrightarrow C \quad \text { is an arbitrary measurable mapping }
\end{array},\right.
$$

where $0 \leq c_{n} \leq c_{n+1} \leq 1$ and $0 \leq \lim c_{n}=c \leq 1$, for all $n \in \mathbb{N}$.

In particular, if $c_{n}=1$, for all $n \in \mathbb{N}$, the sequence $\left(x_{n}\right)_{n}$ is said to be a random Picard iteration scheme.

Theorem 6. Let $(X,\|\|$.$) be a separable Banach space, C$ be a nonempty closed convex subset of $X$, and $(\Omega, \beta, \mu)$ be a complete probability measure space.
Let $T: \Omega \times C \longrightarrow C$ be a continuous $\left(\psi_{1}, \psi_{2}, \varphi\right)$-weakly contractive random operator. Assume that $\psi_{1}$ is convex. Then, the following two statements hold:

(i) There exists a unique random fixed point $x: \Omega \longrightarrow C$ of $T$

(ii) The random Mann iteration scheme converges strongly almost surely to the unique random fixed point $x$ of $T$

Proof

(i) From Corollary 1, $T$ has a unique random fixed point $x: \Omega \longrightarrow C$. Consider the set $F=\{\omega \in \Omega: T(\omega, x(\omega))=x(\omega)\}$. Then, $\mu(F)=1$.

(ii) Consider the set $A=\{\omega \in \Omega: x \longrightarrow T(\omega, x)$ is continuous on $C$ \}.

Let $E_{x_{1}, x_{2}}$ be the set of elements $\omega \in \Omega$ such that

$$
\psi_{1}\left(\| T\left(\omega, x_{1}(\omega)\right)\right)-T\left(\omega, x_{2}(\omega)\right)\left\|\leq \psi_{2}\right\| x_{1}(\omega)-x_{2}(\omega) \|-\varphi\left(\left\|x_{1}(\omega)-x_{2}(\omega)\right\|\right) .
$$

As stated, $\mu(M)=1$, where $M=\cap\left(E_{x_{n}, x_{n+1}} \cap A \cap F\right)$. Let $\omega \in M$. We claim that, for all $n \in \mathbb{N} \in \mathbb{N}$,

$$
\left\|T\left(\omega, x_{n}(\omega)\right)-T(\omega, x(\omega))\right\| \leq\left\|x_{n}(\omega)-x(\omega)\right\| .
$$

Indeed, let us assume that there exists $n_{0} \in \mathbb{N}$ such that

$$
\left\|x_{n_{0}}(\omega)-x(\omega)\right\|<\left\|T\left(\omega, x_{n_{0}}(\omega)\right)-T(\omega, x(\omega))\right\| .
$$

Since $\psi_{1}$ is increasing, we get

$$
\begin{array}{r}
\psi_{1}\left(\left\|x_{n_{0}}(\omega)-x(\omega)\right\|\right)<\psi_{1}\left(\left\|T\left(\omega, x_{n_{0}}(\omega)\right)-T(\omega, x(\omega))\right\|\right) \\
\leq \psi_{2}\left(\left\|x_{n_{0}}(\omega)-x(\omega)\right\|\right)-\varphi\left(\left\|x_{n_{0}}(\omega)-x(\omega)\right\|\right) .
\end{array}
$$

This is a contradiction, since for each $t>0$, $\psi_{1}(t)-\psi_{2}(t)+\phi(t)>0$. Hence, for all $n \in \mathbb{N}$,

$$
\left\|T\left(\omega, x_{n}(\omega)\right)-T(\omega, x(\omega))\right\| \leq\left\|x_{n}(\omega)-x(\omega)\right\| .
$$

Then, for all $n \in \mathbb{N}$,

$$
\begin{aligned}
& \left\|x_{n+1}(\omega)-x(\omega)\right\|=\left\|T\left(\omega, x_{n}(\omega)\right)-T(\omega, x(\omega))\right\| \\
& \leq\left\|x_{n}(\omega)-x(\omega)\right\| .
\end{aligned}
$$

It follows that the sequence $\left(\left\|x_{n}(\omega)-x(\omega)\right\|\right)_{n}$ is decreasing and consequently there exists $l \geq 0$ such that

$$
\lim _{n \longrightarrow \infty}\left\|x_{n}(\omega)-x(\omega)\right\|=l \text {. }
$$

Since $\left(x_{n}\right)_{n}$ is a random Mann iteration scheme, we have, for all $n \in \mathbb{N}$,

$$
\begin{aligned}
& \left\|x_{n+1}(\omega)-x(\omega)\right\|=\left\|\left(1-c_{n}\right) x_{n}(\omega)+c_{n} T\left(\omega, x_{n}(\omega)\right)-x(\omega)\right\| \\
& =\left\|\left(1-c_{n}\right) x_{n}(\omega)+c_{n} T\left(\omega, x_{n}(\omega)\right)-\left(1-c_{n}\right) x(\omega)-c_{n} x(\omega)\right\| \\
& \leq\left(1-c_{n}\right)\left\|x_{n}(\omega)-x(\omega)\right\|+c_{n}\left\|T\left(\omega, x_{n}(\omega)\right)-x(\omega)\right\| .
\end{aligned}
$$

Then,

$$
\left\|x_{n+1}(\omega)-x(\omega)\right\| \leq\left(1-c_{n}\right)\left\|x_{n}(\omega)-x(\omega)\right\|+c_{n}\left\|T\left(\omega, x_{n}(\omega)\right)-T(\omega, x(\omega))\right\| .
$$

Since $\psi_{1}$ is nondecreasing and convex, 


$$
\begin{aligned}
& \psi_{1}\left(\left\|x_{n+1}(\omega)-x(\omega)\right\|\right) \leq\left(1-c_{n}\right) \psi_{1}\left(\left\|x_{n}(\omega)-x(\omega)\right\|\right)+c_{n} \psi_{1}\left(\left\|T\left(\omega, x_{n}(\omega)\right)-T(\omega, x(\omega))\right\|\right) . \\
& \psi_{1}\left(\left\|x_{n+1}(\omega)-x(\omega)\right\|\right) \leq\left(1-c_{n}\right) \psi_{1}\left(\left\|x_{n}(\omega)-x(\omega)\right\|\right)+c_{n} \psi_{2}\left(\left\|x_{n}(\omega)-x(\omega)\right\|\right) \\
& \quad-c_{n} \varphi\left(\left\|x_{n}(\omega)-x(\omega)\right\|\right) .
\end{aligned}
$$

Then,

By passing to the upper limit, we obtain

$$
\psi_{1}(l) \leq(1-c) \psi_{1}(l)+c \psi_{2}(l)-c \varphi(l) .
$$

Then,

$$
\psi_{1}(l) \leq \psi_{2}(l)-\varphi(l) .
$$

This is a contradiction, since for each $t>0$, $\psi_{1}(t)-\psi_{2}(t)+\phi(t)>0$. Then, $l=0$.

Then, for all $\omega \in M$,

$$
\lim _{n \longrightarrow \infty}\left\|x_{n}(\omega)-x(\omega)\right\|=0 .
$$

Consider the set

$$
L=\left\{\omega \in \Omega: \lim _{n \longrightarrow \infty}\left\|x_{n}(\omega)-x(\omega)\right\|=0\right\} .
$$

Since $M \subseteq L$ and $\mu(M)=1$, then $\mu(L)=1$. This shows that the sequence $\left(x_{n}\right)_{n}$ of the $C$-valued random variable converges strongly almost surely to the unique random fixed point $x$.

\section{Applications to Nonlinear Stochastic Integral Equations System}

In this section, we give an application of Theorem 5 to show the existence and the uniqueness of a solution of a nonlinear stochastic integral equations system (NSIE) presented as follows:

$$
\left\{\begin{array}{l}
x(t ; \omega)=h(t ; \omega)+\int_{\mathbb{R}} k(t, s ; \omega) f(s, y(s ; \omega)) d \lambda(s), \\
(\text { N.S.I.E }) \\
y(t ; \omega)=h(t ; \omega)+\int_{\mathbb{R}} k(t, s ; \omega) g(s, x(s ; \omega)) d \lambda(s),
\end{array}\right.
$$

where we have the following:

(a) $\mathbb{R}$ is the locally compact real space with the usual norm of reals and $\lambda$ is the Lebesgue measure on $\mathbb{R}$

(b) $\omega \in \Omega$, where $\Omega$ is the supporting set of the probability measure space $(\Omega, \beta, \mu)$

(c) For all $t \in \mathbb{R}, x(t ;$.$) and y(t ;$.$) are two unknown$ elements in $\mathscr{R} V(\mathbb{R})$ (d) $h(t ; \omega)$ is the stochastic free term defined for $t \in \mathbb{R}$

(e) $k(t, s ; \omega)$ is the stochastic kernel defined for $t$ and $s$ in $\mathbb{R}$

(f) $f$ and $g$ are two real-valued functions

Remark 2 (see [37]). The topological space $\mathbb{R}$ is the union of a countable family of compact subsets $\left\{K_{n}\right\}$ having the properties that $K_{n} \subset K_{n+1}$ and that for any other compact set in $\mathbb{R}$, there is a $K_{i}$ which contains it.

Let $C\left(\mathbb{R}, L_{2}(\Omega, \beta, \mu)\right)$ be the space of all continuous functions from $\mathbb{R}$ into the space $L_{2}(\Omega, \beta, \mu)$ with the topology of uniform convergence on compact sets of $\mathbb{R}$. Note that $C\left(\mathbb{R}, L_{2}(\Omega, \beta, \mu)\right.$ ) is a locally convex space (see [38]) and so it can be endowed with a topology induced by a countable family of seminorms $\left\{\|.\|_{n}\right\}_{n \in \mathbb{N}}$ defined by $\|x\|_{n}=\sup _{t \in K_{n}} \| x$ $(t ;.) \|_{L_{2}(\Omega, \beta, \mu)}$, for each $n \in \mathbb{N}$ and $x \in C\left(\mathbb{R}, L_{2}(\Omega, \beta, \mu)\right)$.

Here,

$$
|\|x(t ; .)\||_{L_{2}(\Omega, \beta, \mu)}^{2}=\int_{\Omega}|x(t ; \omega)|^{2} d \mu(\omega)<\infty .
$$

Note that furthermore, since $L_{2}(\Omega, \beta, \mu)$ is complete, $C\left(\mathbb{R}, L_{2}(\Omega, \beta, \mu)\right)$ is complete with respect to this topology.

We assume that, for each pair $(t, s)$, $k(t, s ;.) \in L_{\infty}(\Omega, \beta, \mu)$ and denote the norm in $L_{\infty}(\Omega, \beta, \mu)$ by

$$
\|k(t, s ; .)\|=\|k(t, s ; .)\|_{L_{\infty}(\Omega, \beta, \mu)}=\mu-e s s \quad \sup _{\omega \in \Omega} \mid k(t, s ; \omega) .
$$

Also, we suppose the following:

(i) For almost all $s \in \mathbb{R}$, the function $t \longmapsto k(t, s ;$.$) is$ continuous from $\mathbb{R}$ into $L_{\infty}(\Omega, \beta, \mu)$

(ii) The function $s \longmapsto\|k(t, s ;).\| .\|x(s ; .)\|_{L_{2}(\Omega, \beta, \mu)}$ is $\lambda$-integrable, for each $x$ in $C\left(\mathbb{R}, L_{2}(\Omega, \beta, \mu)\right)$ and almost all $t \in \mathbb{R}$

(iii) There exists a real-valued function $G$ defined $\lambda$-a.e. on $\mathbb{R}$, such that $G(s)\|x(s ; .)\|_{L_{2}(\Omega, \beta, \mu)}$ is $\lambda$-integrable and for each pair $(t, s, u) \in \mathbb{R}^{3}$,

$$
\|k(t, u ; .)-k(s, u ; .)\| \mid .\|x(u ; .)\|_{L_{2}(\Omega, \beta, \mu)} \leq G(u)\|x(u ; .)\|_{L_{2}(\Omega, \beta, u)} \quad \lambda-a . e .
$$


Consider a random operator $T$ defined on $\Omega \times C\left(\mathbb{R}, L_{2}(\Omega, \beta, \mu)\right)$ such that, for all $x \in C\left(\mathbb{R}, L_{2}(\Omega, \beta, \mu)\right)$ and $t \in \mathbb{R}$,

$$
T(\omega) x(t ; \omega)=\int_{\mathbb{R}} k(t, s ; \omega) x(s ; \omega) d \lambda(s), \quad \text { a.sin } \Omega .
$$

However, for each $t \in \mathbb{R}$, the function $s \longmapsto\|(t, s ;).\| .\|x(t ; .)\|_{L_{2}(\Omega, \beta, \mu)} \quad$ is $\lambda$-integrable; then, $T(\omega) x(t ;.) \in L_{2}(\Omega, \beta, \mu)$ a.s. From (iii) and by using Lebesgue's dominated convergence theorem, $t \longmapsto T(\omega) x(t ;$. is a continuous in mean square, so $T(\omega) x \in C\left(\mathbb{R}, L_{2}(\Omega, \beta, \mu)\right)$ a.s.

Let $B$ and $D$ be two Banach spaces. The pair $(B, D)$ is said to be admissible with respect to the linear operator $T$ if $T(\omega)(B) \subset D$ a.s.

Lemma 1 (see [39]).

(1) The linear operator $T(\omega)$ is continuous from $C\left(\mathbb{R}, L_{2}(\Omega, \beta, \mu)\right)$ into itself a.s.

(2) If $B, D \subset C\left(\mathbb{R}, L_{2}(\Omega, \beta, \mu)\right)$ are two Banach spaces stronger than the space $C\left(\mathbb{R}, L_{2}(\Omega, \beta, \mu)\right)$ such that $(B, D)$ is admissible with respect to $T(\omega)$, then $T(\omega)$ is continuous from $B$ to $D$ a.s.

Definition 9. By a random solution of NSIE, we will mean a pair of functions $(x, y)$ in $\left(C\left(\mathbb{R}, L_{2}(\Omega, \beta, \mu)\right)\right)^{2}$ which satisfies the two equations of NSIE $\mu$-a.e.

Let $\omega \in \Omega$. We consider the two functions $F, G: C\left(\mathbb{R}, L_{2}(\Omega, \beta, \mu)\right) \longrightarrow C\left(\mathbb{R}, L_{2}(\Omega, \beta, \mu)\right)$ defined, for each $t \in \mathbb{R}$ and $\omega \in \Omega$, by $F x(t ; \omega)=f(t, x(t ; \omega))$ and $G x(t ; \omega)=g(t, x(t ; \omega))$.
Let $\rho$ be a positive real number. Consider the ball is centered on $\widehat{0}$ and the neutral element in $D$ is

$$
M(\rho)=\left\{x \in D:\|x\|_{D} \leq \rho\right\} .
$$

Theorem 7. The following conditions hold:

(1) $B$ and $D$ are Banach spaces stronger than $C\left(\mathbb{R}, L_{2}(\Omega, \beta, \mu)\right)$ so that $(B, D)$ is admissible by respect to the integral operator imposed by (66).

(2) The functions $F$ and $G$ maps from $M(\rho)$ into $B$ and there exists three control functions $\psi_{1}, \psi_{2}$, and $\varphi$ such that for any $x_{1}, x_{2} \in M(\rho)$,

$$
\psi_{1}\left(\left\|F x_{1}-G x_{2}\right\|_{B}\right) \leq \psi_{2}\left(\left\|x_{1}-x_{2}\right\|_{D}\right)-\varphi\left(\left\|x_{1}-x_{2}\right\|_{D}\right) \text {. }
$$

(3) $h \in D$

(4) $c(\omega) \in[0,1[$ a.s. and

$$
\left\{\begin{array}{c}
\|h\|_{D}+c(\omega)\|G \hat{0}\|_{B} \leq \rho(1-c(\omega)) \\
\text { a.s. } \\
\|h\|_{D}+c(\omega)\|F \hat{0}\|_{B} \leq \rho(1-c(\omega))
\end{array}\right.
$$

where the norm of $T(\omega)$ is denoted by $c(\omega)$.

Then, if the above conditions hold, system NSIE admits a unique solution in $(M(\rho))^{2}$.

Proof. Consider the mappings $U, V: \Omega \times M(\rho) \longrightarrow D$ such that for all $x \in M(\rho)$,

$$
\begin{aligned}
& U(\omega) x(t ; \omega)=h(t ; \omega)+\int_{\mathbb{R}} k(t, s ; \omega) f(s, x(s ; \omega)) d \lambda(s) \quad \text { a.s. } \\
& V(\omega) x(t ; \omega)=h(t ; \omega)+\int_{\mathbb{R}} k(t, s ; \omega) g(s, x(s ; \omega)) d \lambda(s) \quad \text { a.s. }
\end{aligned}
$$

Let $x \in M(\rho)$. Since

$$
\begin{aligned}
& \|U(\omega) x\|_{D} \leq\|h\|_{D}+c(\omega)\|F x\|_{B} \quad \text { a.s. } \\
& \leq\|h\|_{D}+c(\omega)\|G \widehat{0}\|_{B}+c((\omega))\|F x-G \widehat{0}\|_{B} \quad \text { a.s. } \\
& \leq\|h\|_{D}+c(\omega)\|G \widehat{0}\|_{B}+c(\omega)\|x\|_{D} \quad \text { a.s. } \\
& \leq\|h\|_{D}+c(\omega)\|G \widehat{0}\|_{B}+c(\omega) \rho \quad \text { a.s. } \\
& \leq \rho(1-c(\omega))+c(\omega) \rho \leq \rho \quad \text { a.s., }
\end{aligned}
$$

then $U(\omega) x \in M(\rho)$ a.s. By the same argument, we prove that $V(\omega) x \in M(\rho)$ a.s.

Let $x_{1}, x_{2} \in M(\rho)$. We have

$$
\left\|U(\omega) x_{1}-V(\omega) x_{2}\right\|_{D} \leq c(\omega)\left\|F x_{1}-G x_{2}\right\|_{B} \leq\left\|F x_{1}-G x_{2}\right\|_{B} \quad \text { a.s. }
$$

Then, 


$$
\begin{gathered}
\psi_{1}\left(\left\|U(\omega) x_{1}-V(\omega) x_{2}\right\|_{D}\right) \leq \psi_{1}\left(\left\|F x_{1}-G x_{2}\right\|_{B}\right) \\
\leq \psi_{1}\left(\left\|x_{1}-x_{2}\right\|_{D}\right)-\varphi\left(\left\|x_{1}-x_{2}\right\|_{D}\right) \text { a.s. }
\end{gathered}
$$

which shows that $U(\omega)$ and $V(\omega)$ are $\left(\psi_{1}, \psi_{2}, \varphi\right)$-weakly contractive mappings almost surely on $M(\rho)$. Then, the two operators $U, V: \Omega \times M(\rho) \longrightarrow M(\rho)$ are $\left(\psi_{1}, \psi_{2}, \varphi\right)$-weakly contractive random operators. Therefore, by Theorem 5 , there is a unique common random fixed point of random operators $U$ and $V$, which is the unique stochastic solution of NSIE. This completes the proof.

\section{Conclusion}

In this paper, we have the following:

Theorem 5 is a random generalization of the main result of [32] in a separable Banach space

Corollary 1 generalizes and improves ([36], Theorem $2.5)$ in the setting of Banach spaces

Corollary 4 is a random version of Banach contraction principle in a separable Banach space

Theorem 6 extends ([36], Theorem 5.3) to $\left(\psi_{1}, \psi_{2}, \phi\right)$-weakly contractive random operators

Theorem 7 shows the utility of our main result in solving a system of nonlinear stochastic integral equations

This work will open the door for other deterministic results that can be randomized, for example, [29].

\section{Data Availability}

No data were used to support this study.

\section{Conflicts of Interest}

The authors declare that there are no conflicts of interest.

\section{References}

[1] N. Mani, "Generalized C $\Psi \beta$ - rational contraction and fixed point theorem with application to second order deferential equation $C_{\beta}^{\psi}$-rational contraction and fixed point theorem with application to second order deferential equation," Mathematica Moravica, vol. 22, no. 1, pp. 43-54, 2018.

[2] M. Nazam and M. Arshad, "On a fixed point theorem with application to integral equations," International Journal of Analytic, vol. 7, 2016.

[3] H. Piri, S. Rahrovi, H. Marasi, and P. Kumam, "A fixed point theorem for F-Khan-contractions on complete metric spaces and application to integral equations," The Journal of Nonlinear Sciences and Applications, vol. 10, no. 9, pp. 4564-4573, 2017.

[4] H. R. Marasi and H. Aydi, "Existence and uniqueness Results for two-term nonlinear fractional differential Equations via a fixed point technique," Journal of Mathematics, vol. 2021, Article ID 6670176, 7 pages, 2021.

[5] E. Ameer, H. Aydi, H. Işık, M. Nazam, V. Parvaneh, and M. Arshad, "Some existence Results for a System of nonlinear fractional differential equations," Journal of Mathematics, vol. 2020, Article ID 4786053, 17 pages, 2020.
[6] K. Sawangsup, W. Sintunavarat, and A. F. R. L. DE Hierro, "Fixed point theorems for $F_{\mathfrak{R}}$-contractions with applications to solution of nonlinear matrix equations," Journal Of Fixed Point Theory And Applications, vol. 19, no. No 3, pp. 17111725, 2017.

[7] A. Špaček, Zufällige Gleichungen, Czechoslovak Mathematical Journal, vol. 5, no. No. 4, pp. 462-466, 1955.

[8] O. Hanš, "Reduzierende zufällige transformationen," Czechoslovak Mathematical Journal, vol. 7, no. 1, pp. 154-158, 1957.

[9] O. Hanš, "Random operator equations," in Proceedings of the Fourth Berkeley Symposium on Mathematical Statistics and Probability, pp. 185-202, Los Angels, CL, USA, March 1961.

[10] A. T. Bharucha-Reid, "Fixed point theorems in probabilistic analysis," Bulletin Of the American Mathematical Society, vol. 82, no. 5, pp. 641-657, 1976.

[11] S. Itoh, "Random fixed point theorems with an application to random differential equations in Banach spaces," Journal of Mathematical Analysis and Applications, vol. 67, no. 2, pp. 261-273, 1979.

[12] I. Beg and N. Shahzad, "Random fixed points of random multivalued operators on Polish spaces," Nonlinear Analysis: Theory, Methods \& Applications, vol. 20, no. 7, pp. 835-847, 1993.

[13] D. Dey and M. Saha, "Application of random fixed point theorems in solving nonlinear stochastic integral equation of the Hammerstein type," Malaya Journal of Matematik, vol. 2, pp. 54-59, 2003.

[14] M. Saha, "On some random fixed points of mappings over a Banach space with a probability measure," Proceedings- $\mathrm{Na}$ tional Academy of Sciences india Section A, vol. 76, no. 3, p. 219, 2006.

[15] M. Saha and A. Ganguly, "Random fixed point theorem on a Ćirić-type contractive mapping and its consequence," Fixed Point Theory and Applications, vol. 2012, no. 1, p. 209, 2012.

[16] M. Saha and D. Dey, "Some random fixed point theorems for $(\theta$, L)-weak contractions," Hacett. J. Math. Statist, vol. 41, no. 6, pp. 795-812, 2012.

[17] K. S. Eke, H. Akewe, and S. A. Bishop, "On random fixed point theorems with applications to integral equations," Heliyon, vol. 5, no. 5, Article ID e01641, 2019.

[18] E. Getmanova and V. Obukhovskii, "A note on random equilibrium points of two multivalued maps," Journal of Nonlinear and Variational Analysis, vol. 2, no. 3, pp. 269-272, 2018.

[19] E. Getmanova, V. Obukhovskii, and J. C. Yao, "A random topological fixed point index for a class of multivalued maps," Application Set-Valued Analysis of Optimisation, vol. 1, pp. 95-103, 2019.

[20] J. R. Graef, J. Henderson, and A. Ouahab, "Some Krasnosel'skii type random fixed point theorems," Journal Of Nonlinear Functional Analysis, vol. 2017, pp. 1-34, Article ID 46, 2017.

[21] A. R. Khan, N. Hussain, N. Yasmin, and N. Shafqat, "Random coincidence point results for weakly increasing functions in partially ordered metric spaces," Bulletin Of the Iranian Mathematical Society, vol. 41, no. 2, pp. 407-422, 2015.

[22] G. A. Okeke, S. A. Bishop, and H. Akewe, "Random fixed point theorems in Banach spaces applied to a random nonlinear integral equation of the Hammerstein type," Fixed Point Theory and Applications, vol. 2019, no. 1, pp. 1-24, 2019.

[23] R. A. Rashwan and D. M. Albaqeri, "A common random fixed point theorem and application to random integral equations," 
International Journal Of Applied Mathematics Research, vol. 3, no. 1, pp. 71-80, 2014.

[24] P. Saipara, P. Kumam, and Y. J. Cho, "Random fixed point theorems for Hardy-Rogers self-random operators with applications to random integral equations," Stochastics, vol. 90, no. 2, pp. 297-311, 2018.

[25] P. Saipara, P. Kumam, A. Sombat, A. Padcharoen, and W. Kumam, "Stochastic fixed point theorems for a random Z-contraction in a complete probability measure space with application to non-linear stochastic integral equations," Mathematics in Natural Science, vol. 01, no. 01, pp. 40-48, 2017.

[26] S. Banach, "Sur les opérations dans les ensembles abstraits et leur application aux équations intégrales," Fundamenta Mathematicae, vol. 3, no. 1, pp. 133-181, 1922.

[27] B. E. Rhoades, "Some theorems on weakly contractive maps," Nonlinear Analysis: Theory, Methods \& Applications, vol. 47, no. 4, pp. 2683-2693, 2001.

[28] K. Chaira, A. Eladraoui, and M. Kabil, "Extensions of some fixed point theorems for weak-contraction mappings in partially ordered modular metric spaces," Iranian Journal of Mathematical Sciences And Informatics, vol. 15, no. 1, pp. 111-124, 2020.

[29] L. Ciric, H. Nawab, and C. Nenad, "Common fixed points for Ciric type f-weak contraction with applications," Publicationes Mathematicae Debrecen, vol. 76, no. 1, pp. 31-49, 2010.

[30] V. Gupta and N. Mani, "Common fixed Point for two selfmaps Satisfying a Generalized (Tex translation failed) weakly contractive Condition of integral type," International Journal Of Nonlinear Science, vol. 16, no. 1, pp. 64-71, 2013.

[31] V. Gupta, R. Deep, and A. K. Tripathi, "Existence of coincidence point for weakly increasing mappings satisfies $(\psi$, $\varphi)$-weakly contractive condition in partially ordered metric $\operatorname{spaces}(\psi, \varphi)$-weakly contractive condition in partially ordered metric spaces," International Journal of Computing Science And Mathematics, vol. 7, no. 6, pp. 495-508, 2016.

[32] M. Eslamian and A. Abkar, "A fixed point theorems for generalized weakly contractive mappings in complete metric space," Italian Journal of Pure and Applied Mathematics, vol. 13, 2009.

[33] A. T. Bharucha-Reid, Random Integral Equations, Academic Press, New York, NY, USA, 1972.

[34] M. C. Joshi and R. K Bose, Some Topics in Nonlinear Functional Analysis, John Wiley \& Sons, Hoboken, NJ, USA, 1985.

[35] B. S. Choudhury, "Random Mann iteration scheme," Applied Mathematics Letters, vol. 16, no. 1, pp. 93-96, 2003.

[36] I. Beg and M. Abbas, "Iterative procedures for solutions of random operator equations in Banach spaces," Journal of Mathematical Analysis and Applications, vol. 315, no. 1, pp. 181-201, 2006.

[37] R. F. Arens, "A topology for spaces of transformations," The Annals of Mathematics, vol. 47, no. 3, pp. 480-495, 1946.

[38] K. Yosida, Functional Analysis, Die Grundlehren der Math. Wissenschaften, Band 123, Springer-Verlag, Berlin, Germany, 1965.

[39] W. J. Padgett, "On a nonlinear stochastic integral equation of the Hammerstein type," Proceedings Of the American Mathematical Society, vol. 38, no. 3, pp. 625-631, 1973. 